\title{
Especialización manufacturera y aglomeración urbana en las grandes ciudades de México
}

\author{
Jorge Eduardo Mendoza Cota*
}

\begin{abstract}
This paper uses the concepts of specialisation and urbanisation economies to study the growth of employment in the manufacturing sector in the largest cities of Mexico. We observe a relative decrease in the concentration of industries in the center of the country and a more dynamical increase in the cities of the north of Mexico. We applied three econometric models to estimate the effect of specialisation and urbanisation economies in the main urban areas of Mexico. The results reveal that specialisation economies have had a positive effect in the employment growth. In the areas where manufacturing is more important (Mexico City, Monterrey and Guadalajara) the size of the labour market was a very important factor. Whereas in the case of urbanisation economies, they had a positive but not conclusive effect in the employment growth.
\end{abstract}

Keywords: regional economy, development and growth analysis, industrial studies, industrialization.

\section{Resumen}

En el trabajo se retoman los conceptos de economías de especialización y de urbanización, con el fin de analizar el crecimiento del empleo manufacturero de las principales ciudades de México. En el estudio se aprecia una declinación relativa de la concentración de las industrias en las ciudades con mayor industrialización en la región centro del país y un crecimiento más dinámico del empleo manufacturero en algunas ciudades de la región del norte de México. Para estimar el efecto de las economías de especialización y de urbanización se aplicaron tres modelos econométricos a una base de datos de corte transversal de las ramas industriales al nivel de cuatro dígitos, para las principales áreas metropolitanas y urbanas de México. Los resultados corroboran que la especialización entre industrias ha mostrado un efecto positivo para el crecimiento del empleo manufacturero. En el caso de las áreas urbanas de mayor peso manufacturero (ciudad de México, Monterrey y Guadalajara), el tamaño de los mercados laborales se erigió como un factor determinante del crecimiento del empleo. Respecto del impacto de las economías de urbanización, los resultados muestran un efecto positivo, pero no concluyente, en el crecimiento del empleo manufacturero.

Palabras clave: economía regional, análisis del crecimiento y desarrollo, estudios industriales, industrialización.

* El Colegio de la Frontera Norte. Correo-e: emendoza@colef.mx 


\section{Introducción ${ }^{1}$}

El presente estudio busca analizar los efectos de la especialización manufacturera y de la urbanización, representada por el nivel de población de las ciudades más grandes de México, en el crecimiento manufacturero. Con el fin de lograr este objetivo, se retoma la metodología aplicada para el caso de las ciudades de la frontera norte aplicado por Mendoza (2002) y para las regiones de México por Hanson (1994). Se considera que la importancia y el peso relativo de la actividad manufacturera de los grandes centros urbanos del país hacen necesario explicar su dinámica a la luz de los cambios recientes en la composición regional del crecimiento manufacturero de México.

En efecto, la estructura y dinámica de crecimiento de las industrias manufactureras de las principales ciudades mexicanas (ciudad de México, Guadalajara y Monterrey), experimentaron cambios importantes durante la década de los noventa. Estas transformaciones se relacionaron con la internacionalización de los procesos productivos manufactureros -observados desde finales de la década de los ochenta- y la consecuente reestructuración regional de la dinámica del sector manufacturero del país.

En el caso de la ciudad de México, las industrias relacionadas con el mercado interno continuaron mostrando una mayor especialización con respecto a otros centros urbanos, en un contexto de una menor dinámica de crecimiento del sector manufacturero. En el caso de Guadalajara y Monterrey se aprecia, por un lado, el desarrollo de industrias ligadas a la reestructuración de los procesos productivos en el ámbito internacional y, por otra parte, la permanencia de industrias ligadas al mercado interno.

Debido al mayor tamaño relativo de las ciudades mencionadas y al elevado peso específico de las actividades manufactureras, el trabajo de investigación está enfocado a investigar el impacto que han tenido, por una parte, la localización de las industrias manufactureras en el desarrollo de los nuevos centros urbanos manufactureros y, por otra parte, los efectos de la urbanización en el dinamismo de las manufacturas de las áreas metropolitanas.

El presente estudio se estructura de la siguiente manera: en el inciso 2 se describe el enfoque teórico que sirve de marco con-

${ }^{1}$ Este estudio forma parte de un proyecto de investigación apoyado con financiamiento del Consejo Nacional de Ciencia y Tecnología de México (Conacyt). 
ceptual para explicar las transformaciones del sector manufacturero. En el inciso 3 se analiza el desarrollo reciente de las manufacturas urbanas en las grandes ciudades de México, y se resaltan los principales cambios ocurridos. En el inciso 4 se presentan las variables que conforman el modelo para estimar el impacto de los determinantes locales del crecimiento manufacturero urbano. En el inciso 5 se presentan los resultados de las estimaciones econométricas, y en el último apartado se presentan las conclusiones del estudio.

\section{Externalidades y crecimiento del empleo manufacturero urbano}

\subsection{Especialización industrial, aglomeración urbana y crecimiento manufacturero}

Como se manifestó anteriormente, este estudio busca explicar la nueva geografía económica del país, a partir de evaluar el impacto de la aglomeración urbana y la especialización manufacturera en las principales áreas metropolitanas de México. En esta perspectiva el análisis retoma, como punto de partida inicial, la propuesta de Marshall (1920) que resalta la importancia de las ventajas locales derivadas de las economías externas a la empresa e internas al distrito industrial o región (Becatinni, 1998). Para el caso de la economía mexicana, algunos trabajos se han basado en esta perspectiva para analizar los casos de las industrias de León (Brown y Domínguez, 1997) y Saltillo (Mendoza, 1999). Dentro de esta visión se considera que uno de los factores fundamentales para el crecimiento económico regional es la aglomeración local de las empresas.

Adicionalmente, algunos estudios sobre el funcionamiento de la localización industrial han vinculado los procesos de producción, innovación tecnológica y la organización empresarial al agrupamiento de empresas de un mismo sector en clusters. Este tipo de aglomeración regional permite aprovechar las tecnologías de punta, las técnicas de producción flexible y los nuevos métodos empresariales (just in time), los cuales se han reflejado en un escalamiento industrial (Carrillo y Hualde, 2000).

En la presente investigación se asume que, en el contexto de la creciente integración de las manufacturas mexicanas al proceso de globalización económica, las tendencias a la aglomeración industrial en las áreas urbanas de México tienen una importan- 
cia decisiva para entender los factores de localización urbana de la industria. Esto es así porque la aglomeración da lugar al surgimiento de ventajas regionales derivadas de economías externas de especialización y, en su caso, de economías de urbanización.

En esta perspectiva, resulta indispensable investigar el efecto de las economías de aglomeración en el crecimiento del sector manufacturero de los grandes centros metropolitanos con elevada atracción de actividades económicas y de población. Ahora bien, el crecimiento manufacturero puede explicarse por varios factores, entre los que destacan la existencia de las economías de escala y las ventajas comparativas. A su vez, el concepto de economías de aglomeración permite explicar el crecimiento urbano desde la perspectiva del desarrollo de las externalidades regionales.

En lo que toca a las externalidades derivadas de la especialización manufacturera, algunos autores (Arrow, 1962 y Romer, 1986), retomando la perspectiva de análisis regional de Marshall (1920), han subrayado que la especialización industrial, en un contexto de concentración de mercado, puede generar economías externas derivadas de la difusión tecnológica. Por otra parte, Jacobs (1969) destaca que también, en el contexto de una estructura de mercado competitiva, el desarrollo y la difusión tecnológica surge de la diversidad industrial en una región.

Las externalidades de urbanización se relacionan con el impacto positivo derivado de la diversidad económica y la movilidad de los factores de la producción (trabajo y capital). Sin embargo, el crecimiento de una ciudad implica el aumento de costos de transporte, derivados de la congestión y la mayor distancia para desplazarse de las áreas habitacionales a los centros de producción. Para los trabajadores urbanos, el mayor costo promedio de transporte, el costo de la habitación y el salario urbano son determinantes de la toma de decisiones de localización y demanda de empleo. Por lo que toca a los inversionistas, la maximización de ganancias y la minimización de costos de producción, en un contexto de ventajas relacionadas con la proximidad a los mercados de factores de la producción y bienes finales, son los determinantes básicos a considerar (Henderson, 1974). 


\subsection{Economías de aglomeración, decisiones de localización y crecimiento manufacturero}

La existencia del vínculo entre la especialización, la urbanización y el crecimiento manufacturero de las ciudades de México parte del enfoque de externalidades y su relación con las decisiones de localización regional de la producción manufacturera. En esta perspectiva se considera que el crecimiento manufacturero se relaciona con el impacto que tiene el grado de especialización manufacturera y el crecimiento urbano de las ciudades en las decisiones de localización de las plantas manufactureras en México.

Así, de acuerdo con Bartik (1985), el factor determinante en las decisiones de localización de plantas manufactureras es el nivel de ganancias y, por tanto, de las ventajas regionales o urbanas que pueda presentar un centro urbano con respecto a los demás. En esta perspectiva, en estudios previos se ha detectado que las empresas responden a diversos incentivos existentes en las localidades específicas (Carlton, 1983). Entre éstos, destaca el tamaño de las plantas, los salarios, los costos de energía, los impuestos, los programas de incentivos locales y las variables que miden las economías de aglomeración. Así, los determinantes de la localización, que influyen en la rentabilidad de las regiones, pueden ser agrupados en: factores que afectan la accesibilidad a mercados, factores que impactan los costos de las empresas y factores relacionados con las políticas públicas (Tamayo, 2000)

En esta perspectiva, el presente estudio considera que las ventajas de localización para las empresas manufactureras son influidas por las economías externas y su impacto en la rentabilidad de las industrias localizadas en las áreas urbanas.

Asimismo, se retoma el planteamiento de Marshall respecto de la especialización de los mercados laborales locales o regionales, la disponibilidad de insumos y, en menor medida para el caso de la industria manufacturera mexicana, de la difusión tecnológica. Desde esta perspectiva, las externalidades son desarrolladas cuando alguna de estas condiciones se hace presente en un área urbana. En el caso de la economía mexicana, la globalización de la producción manufacturera y la existencia de economías externas permiten que en las áreas urbanas se fomente el crecimiento de la inversión y el empleo de algunas industrias manufactureras con mayor especialización. 
De esta manera, el enfoque teórico para el análisis del crecimiento y los cambios en la dinámica de la industria manufacturera urbana en México asume que las decisiones de localización que determinan el crecimiento del empleo manufacturero son función de los niveles salariales urbanos, los cambios tecnológicos y las externalidades generadas por la especialización y la aglomeración urbana. El impacto de la aglomeración urbana y manufacturera está relacionado con las ventajas de localización que se reflejan en el nivel de ganancia de las empresas (como consecuencia de la reducción de los costos unitarios).

Cabe destacar que el trabajo empírico de medición del impacto de las externalidades, que generan beneficios relacionados al entorno geográfico de las empresas, presenta dificultades debido a que la estimación de una función de producción para las distintas industrias plantea problemas metodológicos para medir los factores de producción (en particular el capital). Una forma de evaluar la relación de causalidad entre las externalidades derivadas de la especialización de industria y la aglomeración urbana es mediante el establecimiento de un modelo empírico donde el crecimiento del empleo manufacturero sea función de los efectos de la aglomeración urbana. La obtención de coeficientes positivos y significativos estadísticamente estaría demostrando que la existencia de las externalidades reduce los costos unitarios de producción del trabajo, es decir, incrementa la productividad de ese factor.

En la presente investigación, el objetivo fundamental es conocer el impacto de las externalidades en el crecimiento manufacturero de los grandes centros urbanos de México, utilizando como variable el crecimiento del empleo manufacturero. Por tanto, el enfoque basado en una función de producción debe representar específicamente la relación entre externalidades derivadas de la especialización manufacturera y la aglomeración urbana, y el crecimiento de la industria. Para ello es necesario asumir que la función de producción tiene rendimientos de escala crecientes derivados de un impacto positivo de la localización de las empresas. La presentación formal de esta conceptuación teórica requiere que la función de ganancia de corto plazo con rendimientos crecientes:

$$
\Pi=p \mathrm{f}(\mathrm{A}, \mathrm{L})-\mathrm{sL} \text { donde } \mathrm{A}=\mathrm{A}(\mathrm{e}, \mathrm{a}, \mathrm{d}, \mathrm{t})
$$

En la que $p$ son los precios de los bienes de esa industria o empresa, $s$ son los costos salariales, $L$ es la cantidad de trabajo y $A$ 
es igual al cambio tecnológico de las empresas en la región. El enfoque teórico considera que la tecnología existente al nivel regional (urbano) es función del nivel tecnológico nacional y de un vector de variables relacionado con las economías de especialización (e), las economías de aglomeración urbana (a), la diversidad industrial (d) y el tamaño de la planta (t), entre otros.

De esta manera, se puede interpretar que las ventajas de aglomeración se reflejan en el nivel de ganancias de las empresas, mediante la reducción de costos que se derivan de la especialización y las economías de escala (Tamayo, 2000), por lo que el crecimiento del empleo manufacturero se explica no sólo por el avance tecnológico o del conocimiento, sino también de las ventajas locales generadas por la especialización y la aglomeración.

A partir de estos antecedentes teóricos y de análisis empírico, el presente estudio se orienta con base en las siguientes hipótesis:

1. La globalización económica ha impulsado el desplazamiento de procesos de producción manufacturera hacia economías menos desarrolladas. Este fenómeno ha intensificado la aglomeración industrial en algunas áreas urbanas en el norte de México y la especialización de ciertas actividades manufactureras en algunas de ellas.

2. La especialización productiva en una región urbana tiende a generar externalidades a las industrias que se localizan en esa área metropolitana, lo que incentiva la localización de las empresas manufactureras, debido a la presencia de mercados de insumos especializados, y en particular a la integración de mercados laborales con fuerza de trabajo mejor calificada.

3. El crecimiento de las áreas metropolitanas posibilita la presencia de economías de urbanización relacionadas con la diversidad de la actividad económica, los costos de transporte al interior de los grandes centros industriales urbanos y la cercanía a los mercados de factores de producción y de bienes finales.

4. Existen industrias que tienden a 'aglomerarse' en una región aun en ausencia de economías externas, con la finalidad de aprovechar los vínculos 'hacia atrás' y 'hacia adelante' con las empresas que sí están experimentando estas ventajas de localización. Esta relación de causalidad se presenta con mayor fuerza en las grandes ciudades de México. 
A partir de este enfoque, el objetivo de la investigación es evaluar la presencia y el impacto de la localización de las industrias manufactureras en la evolución del empleo de ese sector en las principales áreas urbanas metropolitanas de México. En particular, se busca evaluar a las áreas industriales urbanas más grandes del país como son: La ciudad de México, Guadalajara y Monterrey.

\section{Desarrollo manufacturero urbano en México}

La discusión teórica sobre el desarrollo económico regional en México se ha caracterizado por evidenciar un reducido análisis sobre las causas que determinan la concentración de la industria manufacturera en las grandes áreas urbanas. Recientemente algunos investigadores enfocados al análisis económico regional han argumentado la existencia de una relación entre las políticas comerciales de los países en desarrollo y el crecimiento de los grandes centros urbanos (Krugman y Livas, 1992).

Como ha sido documentado en el estudio de Graizbord y Ruiz (1999), los actuales procesos de urbanización y crecimiento en México se iniciaron alrededor de la década de los cincuenta. Esta etapa de crecimiento inicial se caracterizó por una creciente migración rural y urbana y una mayor participación de la producción manufacturera en el total de actividades económicas. Asimismo, en ese periodo tanto la ciudad de México como Monterrey experimentaron una creciente participación en el empleo manufacturero, siendo beneficiadas por una gran captación de inversión privada y pública que permitió el desarrollo de infraestructura física.

Así pues, se considera que la economía mexicana se caracterizó por tener un patrón de crecimiento urbano concentrado, que generó una demanda interna predominantemente urbana, y que derivó en una rápida industrialización basada en una política de sustitución de importaciones. En este sentido, se argumenta que la concentración de las actividades manufactureras estuvo relacionada tanto con los vínculos 'hacia adelante', que permitieron localizar a las industrias cerca de los mercados de consumidores localizados en los grandes centros urbanos, como con los vínculos 'hacia atrás' que permitieron a las empresas acceder a insumos de otras empresas localizadas en las mismas áreas urbanas. 
De acuerdo con Livas (1992), el crecimiento de las grandes ciudades de México se caracterizó por ser un proceso de doble causalidad, donde los efectos de la aglomeración generaron el crecimiento de las ciudades, que a su vez impulsó la aglomeración. No obstante, se considera que debido a que la estrategia de industrialización estaba orientada fundamentalmente al mercado interno y éste era relativamente pequeño, las economías de escala eran un factor preponderante en las decisiones de localización de la industria cerca de los grandes centros urbanos.

En esta perspectiva, la actual estrategia de industrialización basada en la dinámica exportadora, implicaría un patrón de crecimiento urbano manufacturero en el que las fuerzas 'centrífugas', tales como deseconomías de urbanización por congestión o contaminación compensarían a las fuerzas 'centrípetas', derivadas de las economías de especialización manufacturera y la aglomeración como las externalidades. A continuación se presenta una descripción de las principales características de las actividades manufactureras de las tres ciudades más importantes en México durante la década de los noventa, con la finalidad de generar elementos de juicio para analizar los efectos de la aglomeración urbana y la especialización manufacturera en el crecimiento reciente de la industria manufacturera urbana durante la década de los noventa.

\subsection{Dinámica de los grandes centros urbanos industriales durante la década de los noventa}

De acuerdo con la información que se presenta en el cuadro 1, entre las diez ciudades con mayor empleo en el sector manufacturero en 1988, las tres más importantes fueron la ciudad de México, Guadalajara y Monterrey. Al respecto, cabe resaltar que las ciudades con mayor empleo manufacturero fueron también las que presentaron mayores niveles de aglomeración urbana, medida por el tamaño de la población.

No obstante, como se aprecia en el cuadro 2, el sector manufacturero del área metropolitana de la ciudad de México mostró una tasa de crecimiento promedio anual del empleo manufacturero durante el periodo $1988-1998$ de $0.55 \%$, relativamente inferior al total del empleo manufacturero nacional de $6.12 \%$. Asimismo, en este periodo ninguna de las industrias con mayor crecimiento, desagregadas a cuatro dígitos estuvo localizada en la ciudad de México (véase el cuadro 3). 


\section{Cuadro 1}

\section{Las diez ciudades con más empleo, 1988-1998}

\begin{tabular}{|c|c|c|c|c|}
\hline & \multicolumn{2}{|c|}{ Empleo } & \multicolumn{2}{|c|}{ Siete industrias más grandes } \\
\hline Ciudad & 1988 & 1998 & 1988 & 1998 \\
\hline $\begin{array}{l}\text { AM }^{*} \text { Cd. } \\
\text { México }\end{array}$ & 771,075 & 814,803 & $\begin{array}{l}\text { Imprentas y editoriales, } \\
\text { fibras blandas, plástico, } \\
\text { vestido, otros productos } \\
\text { metálicos, otras sust. } \\
\text { químicas, automotriz } \\
\end{array}$ & $\begin{array}{l}\text { Imprentas y editoriales, } \\
\text { fibras blandas, plástico, } \\
\text { vestido, otros productos } \\
\text { metálicos, otras sust. } \\
\text { químicas, panadería }\end{array}$ \\
\hline AM Monterrey & 184,031 & 284,112 & $\begin{array}{l}\text { Equipo eléctrico, equipo } \\
\text { para usos generales con o } \\
\text { sin motor eléctrico, vidrio, } \\
\text { otros productos metálicos, } \\
\text { hierro y acero, automotriz, } \\
\text { vestido }\end{array}$ & $\begin{array}{l}\text { Equipo eléctrico, equipo para } \\
\text { usos generales con o sin } \\
\text { motor eléctrico, vidrio, otros } \\
\text { productos metálicos, } \\
\text { automotriz, vestido, plástico }\end{array}$ \\
\hline $\begin{array}{l}\text { AM } \\
\text { Guadalajara }\end{array}$ & 148,592 & 256,508 & \begin{tabular}{|l} 
Calzado, bebidas, otros \\
productos metálicos, \\
plástico, panadería, otros \\
productos alimenticios, \\
muebles no metálicos
\end{tabular} & \begin{tabular}{|l|} 
Calzado, otros productos \\
metálicos, plástico, \\
panadería, muebles no \\
metálicos, máquinas de, \\
cálculo y procesamiento \\
informático, equipo \\
electrónico
\end{tabular} \\
\hline $\begin{array}{l}\text { AU** Cd. } \\
\text { Juárez }\end{array}$ & 108,172 & 235,768 & $\begin{array}{l}\text { Equipo eléctrico, equipo } \\
\text { electrónico, textiles, otras } \\
\text { industrias manufactureras, } \\
\text { aparatos de uso doméstico, } \\
\text { equipo para usos generales, } \\
\text { maquinas de cálculo y } \\
\text { procesamiento informático }\end{array}$ & \begin{tabular}{|l|} 
Equipo eléctrico, equipo \\
electrónico, confección de \\
materiales textiles, aparatos \\
de uso doméstico, máquinas \\
de oficina, cálculo y \\
procesamiento informático, \\
automotriz, vestido \\
\end{tabular} \\
\hline AM Puebla & 74,617 & 112,671 & $\begin{array}{l}\text { Tejido de fibras blandas, } \\
\text { automotriz, tejidos de } \\
\text { punto, panadería, } \\
\text { materiales de arcilla, } \\
\text { bebidas, cemento }\end{array}$ & \begin{tabular}{|l|} 
Tejido de fibras blandas, \\
automotriz, tejidos de punto, \\
panadería, materiales de \\
arcilla, vestido, muebles no \\
metálicos
\end{tabular} \\
\hline AM León & 58,457 & 124,753 & $\begin{array}{l}\text { Calzado, cuero y pieles, } \\
\text { hule, vestido, plástico, } \\
\text { panadería, celulosa y papel }\end{array}$ & $\begin{array}{l}\text { Calzado, cuero y pieles, hule, } \\
\text { vestido, plástico, celulosa y } \\
\text { papel, imprentas y editoriales }\end{array}$ \\
\hline AM Toluca & 46,293 & 66,671 & $\begin{array}{l}\text { Automotriz, bebidas, fibras } \\
\text { blandas, plástico, } \\
\text { panadería, otros productos } \\
\text { metálicos, otros productos } \\
\text { alimenticios }\end{array}$ & $\begin{array}{l}\text { Automotriz, bebidas, hilado } \\
\text { y tejido de fibras blandas, } \\
\text { plástico, panadería, otros } \\
\text { productos, vestido }\end{array}$ \\
\hline $\begin{array}{l}\text { AM } \\
\text { Chihuahua }\end{array}$ & 42,340 & 68,132 & $\begin{array}{l}\text { Equipo eléctrico, equipo } \\
\text { electrónico, vestido, } \\
\text { maquinas de, cálculo y } \\
\text { procesamiento informático, } \\
\text { automotriz, aserradero, } \\
\text { cemento }\end{array}$ & $\begin{array}{l}\text { Equipo eléctrico, equipo } \\
\text { electrónico, prendas de } \\
\text { vestir, automotriz, plástico, } \\
\text { materiales de arcilla, } \\
\text { imprentas y editoriales }\end{array}$ \\
\hline
\end{tabular}

Fuente: Elaboración propia a partir de datos del XIII Censo Industrial 1989, del XIV Censo Industrial 1994 y del Censo Económico 1999 (Enumeración integral, resultados oportunos, INEGI). *AM: área metropolitana *AU: área urbana. 


\section{Cuadro 2 \\ Crecimiento manufacturero de las principales ciudades de México}

\begin{tabular}{|l|c|l|l|}
\hline \multicolumn{1}{|c|}{ Ciudad } & Empleo 1988 & Empleo 1998 & TCPA* \\
\hline Nacional & $2,284,133$ & $4,213,566$ & $6.12 \%$ \\
\hline Guadalajara & 148,592 & 256,508 & $5.46 \%$ \\
\hline Ciudad de México & 771,075 & 814,803 & $0.55 \%$ \\
\hline Monterrey & 184,031 & 284,112 & $4.34 \%$ \\
\hline Tijuana & 41,872 & 146,634 & $12.53 \%$ \\
\hline Ciudad Juárez & 108,172 & 235,768 & $7.79 \%$ \\
\hline Puebla & 74,617 & 112,671 & $4.12 \%$ \\
\hline
\end{tabular}

Fuente: Elaboración propia a partir de datos del Censo Industrial de 1988 y del Censo Industrial de 1998, INEGI. "TCPA: tasa de crecimiento promedio anual.

\section{Cuadro 3 \\ Las diez industrias-ciudad con crecimiento más rápido, 1988-1998}

\begin{tabular}{|c|c|c|c|c|c|}
\hline Area & Rama & Descripción & $\begin{array}{c}\text { Empleo } \\
89\end{array}$ & $\begin{array}{c}\text { Empleo } \\
99\end{array}$ & TCPA* \\
\hline Monterrey & 3513 & $\begin{array}{l}\text { Industria de las fibras } \\
\text { artificiales y/o sintéticas }\end{array}$ & 4 & 3,016 & $66.25 \%$ \\
\hline Matamoros & 3213 & $\begin{array}{l}\text { Confección de materiales } \\
\text { textiles }\end{array}$ & 4 & 1,822 & $61.21 \%$ \\
\hline Hermosillo & 3832 & $\begin{array}{l}\text { Fabricación de equipo } \\
\text { electrónico, de radio, televisión, } \\
\text { comunicaciones y científico }\end{array}$ & 11 & 4,517 & $60.18 \%$ \\
\hline Tlaxcala & 3130 & Industria de las bebidas & 4 & 1,218 & $57.19 \%$ \\
\hline Zacatecas & 3240 & Industria del calzado & 3 & 703 & $54.57 \%$ \\
\hline Hermosillo & 3831 & $\begin{array}{l}\text { Fabricación de maquinaria, } \\
\text { equipo, accesorios y } \\
\text { suministros eléctricos }\end{array}$ & 12 & 2,233 & $52.26 \%$ \\
\hline Nuevo Laredo & 3213 & $\begin{array}{l}\text { Confección de materiales } \\
\text { textiles }\end{array}$ & 2 & 361 & $51.96 \%$ \\
\hline Saltillo & 3611 & Alfarería y cerámica & 7 & 1,044 & $50.05 \%$ \\
\hline Monclova & 3220 & Confección de prendas de vestir & 30 & 4,124 & $49.23 \%$ \\
\hline Saltillo & 3410 & $\begin{array}{l}\text { Manufactura de celulosa, papel } \\
\text { y sus productos }\end{array}$ & 7 & 959 & $49.20 \%$ \\
\hline
\end{tabular}

Fuente: Elaboración propia a partir de datos del XIII Censo Industrial 1989, del XIV Censo Industrial 1994 y del Censo Económico 1999 (Enumeración integral, resultados oportunos, INEGI). *TCPA: tasa de crecimiento promedio anual.

Destaca el hecho de que la participación porcentual en el empleo de estas industrias tradicionales se mantuvo sin grandes transformaciones, por lo que su presencia es constante en el periodo 1988-1998. Así pues, se observa una elevada participación, en el 
total de la producción manufacturera en esas ciudades, de las industrias de imprentas y editoriales, de hilados, de plástico y de prendas de vestir y, en menor medida, de la industria automotriz, de sustancias químicas y productos metálicos terminados. En conjunto, estas industrias concentraron alrededor de $40 \%$ del empleo en el área metropolitana de la ciudad de México en el periodo (véase el cuadro 4).

\section{Cuadro 4}

Participación porcentual del empleo, área metropolitana de la ciudad de México, 1988-1998

\begin{tabular}{|c|c|c|c|c|c|}
\hline Rama & Descripción & Empleo & $\begin{array}{c}\begin{array}{c}\text { Proporción de } \\
\text { la rama en el } \\
\text { área }\end{array} \\
\end{array}$ & \begin{tabular}{|c|} 
Empleo de la rama \\
en el ámbito \\
nacional
\end{tabular} & \begin{tabular}{|c|} 
Proporción de la \\
rama nacional
\end{tabular} \\
\hline Total & 1989 & 771,075 & $100.00 \%$ & $2,284,133$ & $33.76 \%$ \\
\hline 3420 & $\begin{array}{l}\text { Imprentas, } \\
\text { editoriales e } \\
\text { industrias conexas }\end{array}$ & 48,399 & $6.28 \%$ & 83,326 & $58.08 \%$ \\
\hline 3212 & $\begin{array}{l}\text { Hilado, tejido y } \\
\text { acabado de fibras } \\
\text { blandas }\end{array}$ & 44,685 & $5.80 \%$ & 100,292 & $44.55 \%$ \\
\hline 3560 & $\begin{array}{l}\text { Elaboración de } \\
\text { productos de plástico }\end{array}$ & 44,076 & $5.72 \%$ & 63,069 & $69.89 \%$ \\
\hline 3220 & $\begin{array}{l}\text { Confección de } \\
\text { prendas de vestir }\end{array}$ & 43,657 & $5.66 \%$ & 121,715 & $35.87 \%$ \\
\hline 3814 & $\begin{array}{l}\text { Fabricación de otros } \\
\text { productos metálicos } \\
\text { terminados }\end{array}$ & 40,268 & $5.22 \%$ & 66,790 & $60.29 \%$ \\
\hline 3522 & \begin{tabular}{|l|}
$\begin{array}{l}\text { Fabricación de otras } \\
\text { sustancias químicas }\end{array}$ \\
\end{tabular} & 39,956 & $5.18 \%$ & 45,561 & $87.70 \%$ \\
\hline 3841 & Industria automotriz & 34,916 & $4.53 \%$ & 101,689 & $34.34 \%$ \\
\hline Total & 1999 & 814,803 & $100.00 \%$ & $4,213,566$ & $19.34 \%$ \\
\hline 3420 & $\begin{array}{l}\text { Imprentas, } \\
\text { editoriales e } \\
\text { industrias conexas }\end{array}$ & 64,359 & $7.90 \%$ & 143,858 & $44.74 \%$ \\
\hline 3220 & $\begin{array}{l}\text { Confección de } \\
\text { prendas de vestir }\end{array}$ & 64,250 & $7.89 \%$ & 453,414 & $14.17 \%$ \\
\hline 3560 & $\begin{array}{l}\text { Elaboración de } \\
\text { productos de plástico }\end{array}$ & 50,256 & $6.17 \%$ & 166,884 & $30.11 \%$ \\
\hline 3814 & $\begin{array}{l}\text { Fabricación de otros } \\
\text { productos metálicos } \\
\text { terminados }\end{array}$ & 48,472 & $5.95 \%$ & 136,391 & $35.54 \%$ \\
\hline 3212 & $\begin{array}{l}\text { Hilado, tejido y } \\
\text { acabado de fibras } \\
\text { blandas }\end{array}$ & 42,023 & $5.16 \%$ & 142,940 & $29.40 \%$ \\
\hline 3522 & $\begin{array}{l}\text { Fabricación de otras } \\
\text { sustancias químicas }\end{array}$ & 41,746 & $5.12 \%$ & 80,377 & $51.94 \%$ \\
\hline 3115 & \begin{tabular}{|l} 
Fabricación de \\
productos de \\
panadería
\end{tabular} & 39,965 & $4.90 \%$ & 156,647 & $25.51 \%$ \\
\hline
\end{tabular}

Fuente: Elaboración propia a partir de datos del XIII Censo Industrial 1989, del XIV Censo Industrial 1994 y del Censo Económico 1999 (Enumeración integral, resultados oportunos, INEGI). 
Por lo que respecta al área metropolitana de la ciudad de Monterrey, el comportamiento del crecimiento del empleo manufacturero $(4.34 \%)$ muestra también un menor dinamismo de la producción que la tasa de crecimiento de las manufacturas en el ámbito nacional, y en particular, que otros centros urbanos manufactureros del norte del país como Tijuana (12.53\%) y Ciudad Juárez (7.79\%) (véase el cuadro 2). Cabe mencionar que la participación de las ramas manufactureras más importantes en el total del empleo del área metropolitana de la ciudad se mantuvieron constantes a lo largo del periodo de estudio. Se destacan, por su importancia, la fabricación de otros productos metálicos terminados, la fabricación de maquinaria, equipo, accesorios y suministros eléctricos y la industria automotriz. Asimismo, las industrias de vidrio y productos de vidrio y la de fabricación de maquinaria y equipo para usos generales, con o sin motor, muestran una gran proporción en las ramas respectivas en el ámbito nacional (véase el cuadro 5).

\section{Cuadro 5}

Participación porcentual del empleo, área metropolitana de

\begin{tabular}{|c|c|c|c|c|c|}
\hline Rama & Descripción & Empleo & $\begin{array}{l}\text { Proporcion de } \\
\text { la rama en el } \\
\text { área }\end{array}$ & $\begin{array}{c}\text { en el ámbito } \\
\text { nacional }\end{array}$ & $\begin{array}{l}\text { Proporción de la } \\
\text { rama nacional }\end{array}$ \\
\hline Total & 1989 & 184,031 & $100.00 \%$ & $2,284,133$ & $8.06 \%$ \\
\hline 3831 & $\begin{array}{l}\text { Fabricación de } \\
\text { maquinaria, equipo, } \\
\text { accesorios y } \\
\text { suministros } \\
\text { eléctricos }\end{array}$ & 16,241 & $8.83 \%$ & 149,627 & $10.85 \%$ \\
\hline 3822 & $\begin{array}{l}\text { Fabricación y } \\
\text { reparación de } \\
\text { maquinaria y equipo } \\
\text { para usos generales, } \\
\text { con o sin motor } \\
\text { eléctrico integrado }\end{array}$ & 12,970 & $7.05 \%$ & 63,090 & $20.56 \%$ \\
\hline 3620 & $\begin{array}{l}\text { Fabricación de } \\
\text { vidrio y productos } \\
\text { de vidrio }\end{array}$ & 12,374 & $6.72 \%$ & 26,243 & $47.15 \%$ \\
\hline 3814 & $\begin{array}{l}\text { Fabricación de otros } \\
\text { productos metálicos } \\
\text { terminados }\end{array}$ & 11,150 & $6.06 \%$ & 66,790 & $16.69 \%$ \\
\hline 3710 & $\begin{array}{l}\text { Industria básica del } \\
\text { hierro y el acero }\end{array}$ & 9,982 & $5.42 \%$ & 69,860 & $14.29 \%$ \\
\hline 3841 & Industria automotriz & 8,201 & $4.46 \%$ & 101,689 & $8.06 \%$ \\
\hline 3220 & $\begin{array}{l}\text { Confección de } \\
\text { prendas de vestir }\end{array}$ & 8,011 & $4.35 \%$ & 121,715 & $6.58 \%$ \\
\hline
\end{tabular}


continuación...

Fuente: Elaboración propia a partir de datos del XIII Censo Industrial 1989, del XIV Censo Industrial 1994 y del Censo Económico 1999 (Enumeración integral, resultados oportunos, INEGI).

La participación significativa de estas ramas en la producción manufacturera del área metropolitana de la ciudad de Monterrey ha sido impulsada, en buena medida, por las estrategias empresariales locales ante el proceso de globalización, como es el caso de Vitro y Alfa. Además, por otra parte, en la región se han desarrollado cadenas productivas en el sector de autopartes (partes de automóvil), con el fin de abastecer a la industria automotriz.

Por su parte, la ciudad de Guadalajara, localizada en la región occidental del país, mostró un mayor dinamismo en el crecimiento del empleo manufacturero $(5.46 \%)$ con relación a la ciudad de México y Monterrey (véase el cuadro 2). Destaca que las siete ramas importantes, según su participación porcentual, concentraban poco más de $35 \%$ del empleo de la ciudad a lo largo del periodo y su composición permaneció casi sin cambios entre 1988 y 1993. Asimismo, se aprecia que cinco de las siete ramas más importantes están orientadas a la producción de bienes tradicionales de consumo interno en general: industria de las bebidas, fabricación de productos de panadería, elaboración de otros productos alimenticios para el consumo humano, industria del cal- 
zado y fabricación y reparación de muebles no metálicos (véase el cuadro 6).

\section{Cuadro 6}

Participación porcentual del empleo, área metropolitana de Guadalajara, 1988-1998

\begin{tabular}{|c|c|c|c|c|c|}
\hline Rama & Descripción & Empleo & $\begin{array}{l}\text { Proporción de } \\
\text { la rama en el } \\
\text { área }\end{array}$ & $\begin{array}{c}\text { Empleo de la rama } \\
\text { en el ámbito } \\
\text { nacional }\end{array}$ & $\begin{array}{c}\text { Proporción de la } \\
\text { rama nacional }\end{array}$ \\
\hline Total & 1989 & 148,592 & $100.00 \%$ & $2,284,133$ & $6.51 \%$ \\
\hline 3240 & Industria del calzado & 14,282 & $9.61 \%$ & 64,813 & $22.04 \%$ \\
\hline 3130 & $\begin{array}{l}\text { Industria de las } \\
\text { bebidas }\end{array}$ & 8,142 & $5.48 \%$ & 99,571 & $8.18 \%$ \\
\hline 3814 & $\begin{array}{l}\text { Fabricación de otros } \\
\text { productos metálicos } \\
\text { terminados }\end{array}$ & 7,900 & $5.32 \%$ & 66,790 & $11.83 \%$ \\
\hline 3560 & \begin{tabular}{|l|} 
Elaboración de \\
productos de plástico
\end{tabular} & 7,854 & $5.29 \%$ & 63,069 & $12.45 \%$ \\
\hline 3115 & $\begin{array}{l}\text { Fabricación de } \\
\text { productos de } \\
\text { panadería }\end{array}$ & 6,732 & $4.53 \%$ & 89,573 & $7.52 \%$ \\
\hline 3121 & $\begin{array}{l}\text { Elaboración de otros } \\
\text { productos } \\
\text { alimenticios para el } \\
\text { consumo humano }\end{array}$ & 5,785 & $3.89 \%$ & 30,342 & $19.07 \%$ \\
\hline 3320 & $\begin{array}{l}\text { Fabricación y } \\
\text { reparación de } \\
\text { muebles no } \\
\text { metálicos }\end{array}$ & 5,606 & $3.77 \%$ & 58,597 & $9.57 \%$ \\
\hline Total & 1999 & 256,508 & $100.00 \%$ & $4,213,566$ & $6.09 \%$ \\
\hline 3560 & \begin{tabular}{|l|} 
Elaboración de \\
productos de plástico
\end{tabular} & 15,088 & $5.88 \%$ & 166,884 & $9.04 \%$ \\
\hline 3240 & Industria del calzado & 14,648 & $5.71 \%$ & 114,079 & $12.84 \%$ \\
\hline 3814 & $\begin{array}{l}\text { Fabricación de otros } \\
\text { productos metálicos } \\
\text { terminados }\end{array}$ & 13,641 & $5.32 \%$ & 136,391 & $10.00 \%$ \\
\hline 3823 & \begin{tabular}{|l|} 
Fabricación de \\
máquinas de oficina, \\
cálculo y \\
procesamiento \\
informático \\
\end{tabular} & 11,831 & $4.61 \%$ & 48,719 & $24.28 \%$ \\
\hline 3832 & $\begin{array}{l}\text { Fabricación de } \\
\text { equipo electrónico, } \\
\text { de radio, televisión, } \\
\text { comunicaciones y } \\
\text { científico }\end{array}$ & 11,669 & $4.55 \%$ & 225,905 & $5.17 \%$ \\
\hline 3320 & $\begin{array}{l}\text { Fabricación y } \\
\text { reparación de } \\
\text { muebles no } \\
\text { metálicos }\end{array}$ & 11,000 & $4.29 \%$ & 134,401 & $8.18 \%$ \\
\hline 3115 & Fabricación de & 9,834 & $3.83 \%$ & 156,647 & $6.28 \%$ \\
\hline
\end{tabular}

Fuente: Elaboración propia a partir de datos del XIII Censo Industrial 1989, del XIV Censo Industrial 1994 y del Censo Económico 1999 (Enumeración integral, resultados oportunos, INEGI). 
Sin embargo, es importante resaltar que durante 1998 se experimentaron varios cambios en la composición de las ramas más importantes. En este año aparecieron dos ramas caracterizadas por estar orientadas a la exportación y a la producción de bienes con mayor nivel tecnológico: la fabricación de máquinas de oficina, cálculo y procesamiento informático y la fabricación de equipo electrónico, de radio, televisión, comunicaciones y científico; además, destaca que la rama de elaboración de productos de plástico incrementó su participación, rebasando incluso a la industria del calzado, que se había mantenido en primer lugar hasta 1993.

En el caso de Guadalajara se observa que la dinámica de las ramas manufactureras líderes está relacionada, por un lado, con el establecimiento de plantas industriales de exportación (gran parte bajo el régimen de maquiladora), o en el desarrollo de industrias de origen doméstico que han logrado incorporarse a las cadenas productivas de las empresas multinacionales.

Se puede concluir que los grandes centros urbanos de México mostraron una reducción de su participación en el empleo manufacturero del país. Asimismo, en el caso de la ciudad de México se observa una especialización de las ramas manufactureras orientadas al mercado interno, mientras que, por otro lado, tanto en Monterrey como en Guadalajara se desarrollaron algunas industrias orientadas a la exportación.

\subsection{Cambios en la especialización manufacturera}

En este apartado se analiza la relación entre el grado de especialización de las industrias (a cuatro dígitos) en las ciudades más grandes del país y el grado de dinamismo manufacturero de dichas ciudades. Para realizar este objetivo se retoma un concepto básico en los estudios de las actividades económicas en el ámbito regional: las economías de especialización, las cuales explican el desarrollo de externalidades a las empresas localizadas en un mismo espacio geográfico. La estimación del índice de especialización manufacturera para las ciudades se basó en la siguiente fórmula:

$$
\text { Índice de especialización }=\frac{V_{i c} / V_{t}}{V_{i p} / V_{p}}
$$


En donde $V_{i c}$ es el empleo de la industria en la ciudad; $V_{t}$ es el empleo total de la ciudad; $V_{i p}$ es el empleo de esa industria al nivel nacional, y $V_{p}$ es el total del empleo en el país.

Cabe destacar que la estimación de las economías externas no es directa y presenta limitaciones para su evaluación justa, debido a su cercana relación con las economías de escala que afectan los costos unitarios y el crecimiento de la producción de las empresas. No obstante, la existencia de externalidades se verifica cuando la creciente especialización industrial o la aglomeración urbana es acompañada de mayor dinamismo en las actividades de las industrias. En el caso de las economías derivadas de la especialización (localización) industrial, surgen de la posibilidad de interacción espacial y de las posibilidades de intercambio que genera la 'yuxtaposición' de empresas de características similares, agrupadas en una misma rama o sector industrial.

Al observar los índices de especialización de la ciudad de México, se aprecia que la especialización de las actividades manufactureras, en la década que va de 1988 a 1998, permaneció concentrada en ramas manufactureras orientadas al mercado interno. Este es el caso de las ramas farmacéutica, del vestido y otros químicos que mantuvieron índices de especialización por arriba de la unidad, lo que implica niveles por arriba del promedio nacional (véase el cuadro 7). Cabe destacar que de acuerdo con la tipología sobre los patrones de cambio tecnológico aplicado a la industria mexicana (Dutrénit y Capdeville,1993), estas ramas manufactureras se caracterizan por tener un bajo nivel tecnológico ampliamente difundido y con bajos requerimientos de capital y de habilidades del trabajo. Por lo anterior, es probable que el efecto de la especialización en esas industrias tenga un limitado efecto en la generación de externalidades en esas industrias.

Respecto de la ciudad de Guadalajara, de nueva cuenta las industrias más especializadas se relacionaron con bienes manufactureros destinados al mercado interno. Así, las ramas de aceites comestibles, la del calzado y la del hule presentaron altos índices de especialización con respecto al comportamiento de esas industrias en el conjunto de las actividades manufactureras del país. No obstante, es importante destacar que en el caso de Guadalajara, durante la década analizada, la industria de computadoras tuvo un crecimiento muy dinámico, vinculado con un elevado grado de especialización en esa área urbana. 


\section{Cuadro 7 \\ Índices de localización de las principales industrias manufactureras}

Fuente: Elaboración propia a partir de datos del XIII Censo Industrial 1989, del XIV Censo Industrial 1994 y del Censo Económico 1999 (Enumeración integral, resultados oportunos, INEGI.) *TCPA: tasa de crecimiento promedio anual.

En lo que corresponde a la ciudad de Monterrey, se observa que las industrias más especializadas se concentraron en las ramas de vidrio, materiales para construcción, hierro y acero y maquinaria en general. Como se analizó en el apartado anterior, estas industrias han tenido tradicionalmente un mayor peso en el total de la producción manufacturera en Monterrey. 
En cuanto a la relación entre especialización y crecimiento, se observa que para los casos de las ciudades de México y Guadalajara las industrias que mostraron mayores índices de especialización también mostraron tasas de crecimiento promedio anual superiores a la alcanzada por el total del sector manufacturero en cada una de esas ciudades. Ello sugiere la posibilidad de que la especialización sea uno de los factores que están determinando la dinámica de crecimiento manufacturero urbano en esas ciudades. No obstante, en el caso de Monterrey, las cinco ramas más especializadas no mostraron tasas de crecimiento superiores a la exhibida por el total de la producción manufacturera de esa ciudad e, inclusive, las industrias de hierro y acero, y del tabaco, que han estado localizadas en esa ciudad por largo tiempo, experimentaron tasas de crecimiento negativo en el periodo. Lo anterior probablemente está relacionado con las transformaciones en la estructura manufacturera de esa área urbana, las cuales se han reflejado en el desarrollo de industrias tales como la de equipo eléctrico, la automotriz y la de autopartes.

\section{Aspectos metodológicos del modelo econométrico}

\subsection{Modelo empírico para estimar los determinantes del crecimiento manufacturero urbano}

En general, el análisis del impacto de las externalidades presenta problemas de interpretación y de medida, pues es difícil diferenciar el impacto de las economías de escala (internas a la firma) y de las economías externas en los costos unitarios de las industrias y, por tanto, en el crecimiento de éstas. En el presente estudio se busca identificar la existencia de las ganancias derivadas de las economías de aglomeración urbanas y en las actividades manufactureras, mediante la estimación del impacto que tienen la aglomeración urbana y la especialización industrial en el crecimiento del empleo manufacturero en las diferentes industrias manufactureras localizadas en las áreas urbanas de México.

La metodología para evaluar el impacto de la aglomeración industrial y urbana en el crecimiento de las manufacturas, se basa en tres modelos econométricos aplicados a una base de datos de corte transversal. El modelo inicial para el análisis de los efectos de aglomeración es el siguiente:

$$
\begin{gathered}
\Delta \ln \left(\mathrm{L}_{\mathrm{irt}} / \mathrm{L}_{\mathrm{rt}}\right)=\alpha+\beta_{1} \ln \left(A 1_{i r t}\right)+\beta_{2} \ln \left(A 2_{i r t}\right)+\beta_{3} \ln \left(D_{i r t}\right)+ \\
\beta_{4} \ln \left(R T_{i r t}\right)+\beta_{5} \ln \left(T_{i r t}\right)+\beta_{6} \ln \mathrm{E}+\beta_{7} \ln r+e_{\text {irt }}
\end{gathered}
$$


La variable dependiente es el crecimiento del empleo relativo y está definida como el crecimiento promedio anual del crecimiento del empleo manufacturero de cada industria urbana:

$$
\Delta \ln \left(\mathrm{L}_{\mathrm{irt}} / \mathrm{L}_{\mathrm{rt}}\right)=\left[\ln \left(\mathrm{L}_{\mathrm{irs}}\right)-\ln \left(\mathrm{L}_{\mathrm{irt}}\right)\right]-\left[\ln \left(\mathrm{L}_{\mathrm{is}}\right)-\ln \left(\mathrm{L}_{\mathrm{it}}\right)\right],
$$

Los subíndices $i$ y $r$ expresan la rama industrial y el área urbana, respectivamente; $s$ corresponde al periodo final y $t$ al periodo inicial.

En lo que corresponde a las variables explicativas, el modelo econométrico mide la especialización de la producción manufacturera a través de tres índices que fueron construidos con base en la información sobre las manufacturas desagregadas a cuatro dígitos, de acuerdo con la Clasificación Mexicana de Actividades y Productos (CMAP).

A1 es el índice de especialización industrial que fue construido con base en el modelo desarrollado por Glaeser et al. (1992). El índice representa la fracción del empleo de la industria en el total de las manufacturas urbanas (a cuatro dígitos), normalizada por la fracción del empleo manufacturero urbano en el total del empleo manufacturero nacional. En este estudio se considera que la especialización manufacturera expresada por este índice $(\mathrm{A} 1>1)$ genera economías externas a los establecimientos manufactureros. Su comprobación empírica debe reflejarse en un positivo coeficiente de esta variable.

$$
A 1_{\text {irt }}=\left(\mathrm{L}_{\mathrm{irt}} / \mathrm{L}_{\mathrm{it}}\right) /\left(\mathrm{L}_{\mathrm{it}} / \mathrm{L}_{\mathrm{t}}\right)
$$

A2 constituye la aglomeración con industrias relacionadas, que son aquellas que comparten una clasificación dada al nivel de dos dígitos de la CMAP.

$$
A 2_{i r t}=\left(\mathrm{L}_{\mathrm{krt}} / \mathrm{L}_{\mathrm{irt}}\right) /\left(\mathrm{L}_{\mathrm{kt}} / \mathrm{L}_{\mathrm{it}}\right)
$$

Donde $k$ indica la industria al nivel de dos dígitos. Esta variable es una proxi para estimar los efectos de los vínculos entre los oferentes y demandantes de insumos entre empresas agrupadas en una industria a cuatro dígitos de desagregación. El índice representa la participación del empleo de las industrias a cuatro dígitos en las industrias agrupadas a dos dígitos en un área urbana ponderada por la misma proporción en el ámbito nacional. Mientras más elevado sea el índice, mayor será el nivel de especialización en esa región o área urbana. Es importante resaltar que la estimación del impacto de las externalidades en el creci- 
miento del empleo manufacturero asume un coeficiente positivo para este índice.

$D i$ es el índice de diversificación de la actividad productiva en el área urbana $r$ en la industria $i$, durante el periodo $t$. También se construye al nivel de cuatro dígitos de la CMAP. El índice de diversificación se estima de la siguiente forma: $\Sigma_{\mathrm{i} \neq \mathrm{r}}\left(\mathrm{L}_{\mathrm{ikt}} / \mathrm{L}_{\mathrm{irt}}\right)^{2}$ y después, en el modelo se pondera por el mismo indicador en el ámbito nacional, de tal forma que:

$$
\mathrm{Di}_{\mathrm{irt}}=\Sigma_{\mathrm{k} \neq \mathrm{i}}\left(\mathrm{L}_{\mathrm{krt}} / \mathrm{L}_{\mathrm{rit}}\right)^{2} / \Sigma_{\mathrm{k} \neq \mathrm{i}}\left(\mathrm{L}_{\mathrm{kt}} / \mathrm{L}_{\mathrm{it}}\right)^{2}
$$

Este índice es semejante al índice Herfindahl-Hirschman utilizado en los estudios de organización industrial para medir el grado de concentración industrial. Cuando sólo existe una empresa en la industria, el índice tiene un valor de uno, y tenderá a cero si las empresas se distribuyen de manera uniforme al interior de la industria. En el caso de los estudios regionales, el índice se utiliza para estimar el impacto de las economías externas derivadas de la diversificación en las industrias. Se asume que la presencia de externalidades está asociada positivamente a la diversidad industrial (Jacobs, 1984). Mientras más bajo es el valor del índice (ponderado por la diversidad al nivel nacional), más grande es la diversidad.

Otra variable considerada es $T$, o tamaño medio de los establecimientos manufactureros, la cual es una variable proxi de la estructura de mercado existente en las industrias a cuatro dígitos. Debido a que no todas las empresas operan con el mismo nivel de eficiencia o tecnología, se introduce $T=\left(\mathrm{L}_{\mathrm{irr}} / \mathrm{N}_{\mathrm{irt}}\right) /\left(\mathrm{L}_{\mathrm{it}} /\right.$ $\mathrm{N}_{\mathrm{it}}$ )

$R t$ son las remuneraciones totales del área urbana ponderadas al nivel nacional. $E$ y $r$ representan a las variables de control del año base, empleo y salarios totales del sector manufacturero por industria a cuatro dígitos. En el caso de la primera variable, se asume que el mayor nivel de empleo en las industrias urbanas debería determinar un crecimiento menor en el empleo de esa industria. Por su parte, la segunda variable nos permite considerar los efectos del desplazamiento de los trabajadores de las áreas urbanas de bajos salarios hacia las de salarios más altos.

Finalmente, se utilizan dos modelos con variables dicotómicas para estimar las economías externas de urbanización de las grandes áreas metropolitanas (ciudad de México, Guadalajara y Monterrey), incluyendo adicionalmente a la población urbana como variable proxi para representar las economías de urbani- 
zación. El primer modelo incorpora variables dummy de pendiente, con el fin de evaluar el efecto de cada una de las variables independientes en el crecimiento del empleo manufacturero de las grandes ciudades, tal y como se presenta a continuación: $\Delta \ln \left(\mathrm{L}_{\mathrm{irt}} / \mathrm{L}_{\mathrm{rt}}\right)=\alpha+\beta_{1} \ln \left(A 1_{i r t}\right)+\beta_{2}\left[\ln A 1_{\text {irt }}{ }^{*} \mathrm{df}\right]+\beta_{3} \ln \left(A 2_{i r t}\right)+$

$\beta_{4}\left[\ln A 2_{i r t}{ }^{*} \mathrm{df}\right]+\beta_{5} \ln \left(\mathrm{D}_{\text {irt }}\right)+\beta_{6}\left[\ln D_{\text {irt }}\right.$ "df $]+\beta_{7} \ln \left(\mathrm{RT}_{i r t}\right)+$ $\beta_{8}\left[\operatorname{lnRT_{irt}}{ }^{*} \mathrm{df}\right]+\beta_{9} \ln \left(\mathrm{T}_{i r t}\right) \beta_{10}\left[\ln \mathrm{T}_{i r t}{ }^{*} \mathrm{df}\right]+\beta_{11} \ln \left(\mathrm{EU}_{\mathrm{rt}}\right)+$ $\beta_{12}\left[\ln E U_{\mathrm{rt}}^{*}{ }^{*} \mathrm{df}\right]+\mathrm{e}_{\mathrm{irt}}$

Donde EU son las economías urbanas que son externas a cualquier industria e implican que el uso de los recursos disponibles es más eficiente en las grandes ciudades debido a la existencia de mercados laborales globales o un sector de servicios grande con gran interacción con el sector manufacturero (Henderson, 1974). Por tanto, se asume que mientras más grande es la población de las áreas urbanas, más probable es la existencia de economías de urbanización.

El tercer modelo adiciona una variable dummy de intercepto para verificar si, en conjunto, el empleo manufacturero de las tres ciudades más grandes de México es impactado de diferente manera por las variables de localización y urbanización. El modelo se constituye de la siguiente forma:

$\Delta \ln \left(\mathrm{L}_{\mathrm{irt}} / \mathrm{L}_{\mathrm{rt}}\right)=\alpha+\beta_{1} \ln \left(A 1_{i r t}\right)+\beta_{2} \ln \left(A 2_{i r t}\right)+\beta_{3} \ln \left(D_{i r t}\right)+\beta_{4} \ln \left(\mathrm{RT}_{i r t}\right)$ $+\beta_{5} \ln \left(\mathrm{T}_{i r t}\right)+\beta_{6} \ln \left(\mathrm{EU}_{\mathrm{rt}}\right)+\beta_{7}[\mathrm{Dum}]+\mathrm{e}_{\mathrm{irt}}$

Donde Dum es la variable dummy que se aplica a la ciudad de México, Guadalajara y Monterrey.

\subsection{Base de datos}

La base de datos está constituida por las 54 ramas manufactureras a cuatro dígitos que se publica en los censos industriales. Esta clasificación fue aplicada en 42 áreas metropolitanas y urbanas, compuestas por las ciudades capitales de las entidades del país, la ciudad de México y los principales centros urbanos manufactureros (véase adelante, el cuadro 10). La elaboración de esta base de datos de la industria manufacturera urbana en México se realizó integrando la información manufacturera por municipios, de acuerdo a la clasificación de áreas metropolitanas y urbanas desarrollada por el Instituto Nacional de Estadística, Geografía e Informática (INEGI). 


\section{Evidencia econométrica}

El análisis empírico busca corroborar las hipótesis derivadas del enfoque teórico basado en los conceptos de economías externas y especialización productiva. Cabe destacar que las variables explicativas del modelo econométrico se corrieron individualmente contra la variable dependiente. Los resultados de las regresiones mostraron, en todos los casos, coeficientes estadísticamente significativos, mostrando los signos esperados, con excepción del coeficiente de especialización al interior de la industria.

$\mathrm{Al}$ correr el primer modelo que incluye todas las variables de localización manufacturera y de control, para el periodo 19881993, el coeficiente de la aglomeración entre industrias relacionadas presentó un efecto positivo, y estadísticamente significativo al $1 \%$ de confianza, en el crecimiento del empleo manufacturero en todas las áreas metropolitanas analizadas (véase el cuadro 8).

\section{Cuadro 8 \\ Variable dependiente: crecimiento del empleo manufacturero, 1988-1993}

\begin{tabular}{|l|r|r|r|r|r|r|}
\hline \multicolumn{1}{|c|}{ Variables } & \multicolumn{5}{c|}{ Coeficientes } \\
\hline A1 & -0.21 & & & & & -0.15 \\
\hline & $(-19.20)^{*}$ & & & & & $(-6.71)^{*}$ \\
\hline A2 & & 0.22 & & & & 0.06 \\
\hline DI & & & 0.08 & & & $(2.22)^{* *}$ \\
\hline & & & $(3.08)^{*}$ & & & -0.07 \\
\hline RT & & & & -0.22 & & $(-2.25)^{* *}$ \\
\hline & & & & $(-5.74)^{*}$ & & 0.004 \\
\hline T & & & & & -0.22 & $(0.081)$ \\
\hline & & & & & $(-8.57)^{*}$ & -0.08 \\
\hline E & & & & & & $(-2.39)^{* *}$ \\
\hline & & & & & & 0.07 \\
\hline $\mathrm{R}$ & & & & & & -0.08 \\
\hline & 0.140 & 0.108 & 0.004 & 0.027 & 0.024 & $(-2.15)^{* *}$ \\
\hline $\mathrm{R}^{2}$ ajustada & 1.93 & 1.92 & 1.95 & 1.96 & 1.95 & 0.164 \\
\hline D.W. & 368.69 & 273.35 & 9.26 & 63.41 & 56.65 & 63.42 \\
\hline F-estadístico & & & & & & 1.95 \\
\hline
\end{tabular}

Fuente: Elaboración propia a partir de datos del XIII Censo Industrial 1989, del XIV Censo Industrial 1994 y del Censo Económico 1999 (Enumeración integral, resultados oportunos, INEGI). "Significativos al $1 \%$ de confianza. * Significativos al $5 \%$ de confianza. Ajuste de heterocedasticidad por el método de White. Las pruebas $\mathrm{F}$ rechazan la hipótesis nula al 1\% de confianza. 
Cabe destacar que este índice de aglomeración se construyó como una proxi que busca medir el vínculo comprador-vendedor de insumos, mediante el cálculo de la participación del empleo de las industrias a dos dígitos en el empleo de las industrias a cuatro dígitos, en el ámbito estatal, normalizado por la participación del empleo de las industrias a dos dígitos relativo al empleo industrial a cuatro dígitos, en el ámbito nacional. Asimismo, debe señalarse que algunos autores que han trabajado el tema de externalidades y han utilizado este índice, asumen que mientras mayor sea la aglomeración de industrias relacionadas (dos dígitos) a la industria en cuestión (cuatro dígitos), existirán mayores externalidades positivas para esa industria (Hanson, 1994). Por tanto, el resultado anterior parece corroborar la posible existencia de economías externas, derivadas de la especialización industrial al nivel de subsector manufacturero, que han impulsado el crecimiento del empleo manufacturero.

No obstante, se aprecia que al correr la regresión con todas las variables incluidas en el modelo, los coeficientes de la aglomeración al interior de la industria y del índice de diversidad industrial fueron negativos y estadísticamente significativos. Este resultado sugiere, por una parte, que la especialización de ramas industriales a cuatro dígitos de los censos manufactureros no ha sido un factor determinante del crecimiento del empleo manufacturero. Así, el grado de especialización al nivel más desagregado parece no tener un efecto positivo en el crecimiento, a diferencia de la especialización al nivel más agregado de dos dígitos, lo cual apunta hacia una mayor importancia de los vínculos interindustriales.

En lo que toca al índice de diversidad, cabe señalar que éste ha sido usado para medir el grado de concentración de las industrias. En el contexto urbano o regional, el índice es utilizado para evaluar el impacto de la diversidad de industrias y la existencia de externalidades locales (Jacobs, 1984). Así, los coeficientes estimados sugieren que el factor diversidad no ha jugado un papel preponderante en la estimulación del crecimiento manufacturero.

Por otra parte, el tamaño de la planta exhibió una relación negativa con el crecimiento del empleo $(-0.08)$ y fue estadísticamente significativo al 5\% de confianza. Lo anterior sugiere que en este periodo, los cambios en la dinámica del crecimiento del empleo manufacturero se basan en empresas de menor tamaño relacionadas con el mercado de las áreas urbanas, y probable- 
mente en empresas proveedoras de las grandes firmas de las áreas metropolitanas con mayor crecimiento del empleo. Al parecer, en esta etapa las pequeñas empresas parecen estar ligadas tanto a la dinámica de las empresas orientadas al mercado interno como a las empresas vinculadas con el comercio exterior, en particular las relacionadas con proveedores del sector maquilador.

Asimismo es destacable que el coeficiente de las remuneraciones relativas no fue estadísticamente significativo y presentó un signo positivo. Lo anterior no soporta el supuesto de que los salarios relativos al nivel urbano se correlacionan de manera inversa al crecimiento del salario. No obstante, la variable de control de remuneraciones totales arrojó un coeficiente negativo y estadísticamente significativo, lo que sugiere que en las áreas metropolitanas estudiadas el nivel de remuneraciones totales está correlacionado inversamente con el crecimiento del empleo.

Finalmente, la variable de control que incorpora al nivel total de empleo tuvo un coeficiente positivo, de acuerdo con el supuesto de que la mayor cantidad de empleo manufacturero genera externalidades derivadas de la creación de mercados de trabajo integrados (pooled labor markets), aunque no fue significativo estadísticamente.

En el periodo 1993-1998, de nueva cuenta las regresiones individuales de las variables independientes presentaron coeficientes estadísticamente significativos (véase el cuadro 9). En lo referente a los resultados del modelo que incluye todas las variables, no se observan cambios importantes respecto del impacto de la aglomeración industrial. No obstante, las variables de control presentaron resultados estadísticamente significativos y con los signos esperados. Este comportamiento parece sugerir que la tendencia evidenciada desde 1993 fue constante hasta el año de 1998. Lo anterior corrobora que la aglomeración entre industrias relacionadas a cuatro dígitos de los censos industriales y el tamaño de los mercados laborales, reflejado por la relación positiva entre empleo total al inicio del periodo y el crecimiento del empleo manufacturero, siguen siendo uno de los factores explicativos de la dinámica de los determinantes del empleo manufacturero por áreas metropolitanas en México.

El segundo modelo se basa en un análisis de regresión que incluye a variables dicotómicas. Este método permite diferenciar cada uno de los coeficientes de las variables independientes de los centros urbanos más grandes del país del resto de las variables de todas las áreas urbanas y metropolitanas en conjunto. 
Cuadro 9

Variable dependiente: crecimiento del empleo manufacturero, 1993-1998

\begin{tabular}{|c|c|c|c|c|c|c|}
\hline \multirow{2}{*}{\begin{tabular}{|l}
\multicolumn{2}{|r}{ Variables } \\
A1
\end{tabular}} & \multicolumn{6}{|c|}{ Coeficientes } \\
\hline & -0.16 & & & & & -0.09 \\
\hline & $(-9.90) *$ & & & & & $(-3.19) *$ \\
\hline \multirow[t]{2}{*}{ A2 } & & 0.19 & & & & 0.12 \\
\hline & & $(10.06)^{*}$ & & & & $(3.91) *$ \\
\hline \multirow[t]{2}{*}{ DI } & & & 0.07 & & & -0.02 \\
\hline & & & $(2.14) *$ & & & $(-0.50)$ \\
\hline \multirow[t]{2}{*}{ RT } & & & & -0.091 & & 0.06 \\
\hline & & & & $(-3.02)^{*}$ & & (1.84) \\
\hline \multirow[t]{2}{*}{$\mathrm{T}$} & & & & & -0.09 & -0.02 \\
\hline & & & & & $(-4.22) *$ & $(-0.69)$ \\
\hline \multirow[t]{2}{*}{ E } & & & & & & 0.09 \\
\hline & & & & & & $(2.53) *$ \\
\hline \multirow[t]{2}{*}{$\mathrm{R}$} & & & & & & -0.07 \\
\hline & & & & & & $(-2.75) *$ \\
\hline $\mathrm{R}^{2}$ ajustada & 0.1006 & 0.102 & 0.001 & 0.006 & 0.006 & 0.113 \\
\hline D.W. & 1.93 & 1.92 & 1.95 & 1.95 & 1.94 & 1.92 \\
\hline F-estadístico & 240.95 & 258.18 & 3.92 & 14.99 & 14.93 & 42.19 \\
\hline
\end{tabular}

Fuente: Elaboración propia a partir de datos del XIII Censo Industrial 1989, del XIV Censo Industrial 1994 y del Censo Económico 1999 (Enumeración integral, resultados oportunos, INEGI). *Significativos al 1\% de confianza. Ajuste de heterocedasticidad por el método de White. Las pruebas $\mathrm{F}$ rechazan la hipótesis nula al 1\% de confianza.

Los resultados sugieren que la aglomeración entre industrias a cuatro dígitos del censo industrial de México siguió presentándose como la variable determinante para el total de áreas metropolitanas analizadas (véase el cuadro 10).

No obstante, al introducir los parámetros de las variables dicotómicas, se observa que para el caso de las tres áreas urbanas metropolitanas más grandes, el coeficiente de la variable dummy de las remuneraciones al empleo manufacturero urbano exhibió un signo negativo $(-0.37)$ y significativo. Lo anterior parece sugerir que el patrón de crecimiento del empleo manufacturero en las áreas metropolitanas más grandes de México es diferente del resto del país. Así, la dinámica de la actividad manufacturera de estas ciudades, medida por el crecimiento del empleo, parece conformarse de acuerdo con el supuesto de una relación inversa entre las remuneraciones y el crecimiento del empleo manufacturero. Lo anterior sugiere que los salarios urbanos relativos son un factor determinante en las decisiones de localización de la industria manufacturera. Al parecer, tanto los diferenciales sala- 
Cuadro 10

Variables dicotómicas para las ciudades de México, Guadalajara y Monterrey, 1993-1998

\begin{tabular}{|l|r|r|}
\hline \multicolumn{1}{|c|}{ Variables } & \multicolumn{2}{|c|}{ Coeficientes } \\
\hline & -0.08 & -0.08 \\
\hline A2 & $(-3.02)^{*}$ & $(-2.97)^{*}$ \\
\hline & 0.12 & 0.12 \\
\hline DI & $(3.91)^{*}$ & $(4.06)^{*}$ \\
\hline & -0.01 & 0.01 \\
\hline RT & $(-0.33)$ & $(0.15)$ \\
\hline & 0.06 & 0.05 \\
\hline T & $(1.72)$ & $(1.41)$ \\
\hline & -0.02 & -0.02 \\
\hline P & $(-0.79)$ & $(-0.90)$ \\
\hline & -0.00 & -0.00 \\
\hline A1d & $(-1.17)$ & $(-4.22)^{*}$ \\
\hline & -0.05 & \\
\hline A2d & $(-0.53)$ & \\
\hline & -0.08 & \\
\hline DId & $(-0.58)$ & \\
\hline & 0.35 & \\
\hline RTd & $(1.11)$ & \\
\hline & -0.37 & \\
\hline Td & $(-1.97)$ & \\
\hline & 0.04 & \\
\hline Pd & $(0.61)$ & \\
\hline & 0.00 & \\
\hline Dum & $(0.95)$ & \\
\hline & & 0.21 \\
\hline R $^{2}$ ajustada & 0.11 & 0.11 \\
\hline D.W. & 1.93 & 1.93 \\
\hline F-estadístico & 23.76 & 40.67 \\
\hline & & $(3.10)^{*}$ \\
\hline & & \\
\hline & & \\
\hline & & \\
\hline & & \\
\hline & & \\
\hline & & \\
\hline & &
\end{tabular}

Fuente: Elaboración propia a partir de datos del XIII Censo Industrial 1989, del XIV Censo Industrial 1994 y del Censo Económico 1999 (Enumeración integral, resultados oportunos, INEGI). *Significativos al 1\% de confianza. Ajuste de heterocedasticidad por el método de White. Las pruebas $\mathrm{F}$ rechazan la hipótesis nula al $1 \%$ de confianza. 
riales entre las ciudades de México como la existencia de encadenamientos 'hacia atrás' y 'hacia adelante' derivados de la presencia de mercados de insumos y de demanda final localizados en las grandes ciudades de México han sido factores determinantes del crecimiento manufacturero de los grandes centros urbanos del país durante la década de los noventa.

Cabe destacar que los coeficientes restantes no son estadísticamente significativos, por lo que el parámetro con signo positivo que refleja las economías urbanas, para el caso de las grandes áreas metropolitanas, no es concluyente. Así, el análisis empírico presenta evidencia de que la aglomeración urbana tiene un efecto negativo, pero muy pequeño, para el conjunto de las áreas urbanas incluidas en el análisis econométrico y positivo pero poco significativo para las áreas específicas incluidas en las variables dummies.

Finalmente, el tercer modelo incluye una variable dummy de intercepto para los grandes centros urbanos, y presenta el mismo patrón de comportamiento de las variables de especialización. No obstante, el coeficiente de la variable de población urbana mostró un signo positivo y significativo, lo que implica que el conjunto de áreas urbanas no ha generado economías de urbanización, sino por el contrario, el conjunto de actividades desarrolladas en su interior ha impactado negativamente el crecimiento urbano. Lo anterior probablemente está relacionado con las limitaciones en infraestructura pública urbana y las limitaciones del mercado interno. No obstante, la variable dummy de intercepto para la proxi de economías de urbanización aplicada para las grandes áreas urbanas mostró un signo positivo $(0.21)$ y estadísticamente significativo, lo que parece implicar que en el caso de las ciudades de México, Guadalajara y Monterrey, los efectos de la localización manufacturera y la aglomeración urbana tienen un efecto positivo, debido al tamaño de la población y la existencia de actividades de servicios que permiten generar economías de urbanización a las empresas manufactureras localizadas en estas ciudades.

\section{Conclusiones}

El empleo manufacturero urbano durante la década de los noventa exhibió cambios en la dinámica de crecimiento por ciudades como resultado de la diferenciación regional experimentada en este periodo. Entre los principales factores que explican di- 
chas transformaciones, destaca la creciente especialización de la producción manufacturera en las ciudades más importantes del país. De esta forma, ciudades ubicadas en el norte del país han tendido a especializarse en manufacturas para la exportación, mientras que en las áreas urbanas del centro del país han continuado orientadas predominantemente a la producción para el mercado interno.

Se observó que en la década de los ochenta, la ciudad de México aún concentraba importantes actividades manufactureras con gran peso en el ámbito nacional (imprentas y editoriales, plástico y sustancias químicas). Hacia finales de dicha década, las áreas metropolitanas de Tijuana y Ciudad Juárez desarrollaron industrias con elevada participación del empleo, vinculadas con las actividades de la industria maquiladora de exportación.

Entre 1993 y 1998, las tres áreas metropolitanas más grandes de acuerdo con el empleo manufacturero fueron por orden de importancia: ciudad de México, Monterrey y Guadalajara. Se aprecia también una desaceleración en el ritmo de crecimiento de estas ciudades con respecto al promedio nacional de la industria manufacturera.

Con respecto a los resultados de las diferentes estimaciones econométricas de los efectos dinámicos de la localización industrial y las economías de urbanización en el crecimiento del empleo manufacturero, se observa que el coeficiente de la variable que refleja la aglomeración entre industrias relacionadas fue positivo en los dos periodos estudiados (1988-1993 y 1993-1998). No obstante, las economías externas derivadas de la aglomeración dentro de la misma industria presentaron un efecto negativo en el crecimiento urbano manufacturero. De esta forma, los resultados sugieren que las economías externas vínculo comprador-vendedor de insumos entre industrias relacionadas a dos dígitos parece haberse convertido en un factor dinámico de la actividad manufacturera urbana.

El coeficiente de la variable que representa el tamaño de promedio de los establecimientos industriales mostró un signo negativo, lo que sugiere que el crecimiento del empleo manufacturero está vinculado a un mercado basado en empresas de menor tamaño, relacionadas, por un lado, con la expansión de la industria maquiladora y el desarrollo de cadenas productivas relacionadas con la red de proveedores de empresas relacionadas con el comercio exterior y, por otro lado, con las empresas enfocadas al mercado interno. Finalmente, los coeficientes de la población 
urbana, proxi de las economías estáticas de urbanización, fueron negativos, lo que muestra la presencia de deseconomías externas, derivadas probablemente de los altos costos de transporte (Henderson, 1974).

$\mathrm{Al}$ incluir variables dicotómicas para las áreas urbanas manufactureras más importantes, se obtuvo un coeficiente negativo y estadísticamente significativo para la variable de economías de urbanización, lo cual sugiere que en el caso de las grandes áreas metropolitanas los efectos de la aglomeración urbana no son un factor determinante del crecimiento del empleo manufacturero. No obstante, es importante destacar que el coeficiente de la variable dicotómica (de intercepto) aplicada a las industrias localizadas en las grandes ciudades tuvo un impacto positivo derivado de la especialización manufacturera y la aglomeración urbana de las ciudades.

De esta manera, aunque los resultados del modelo econométrico no son totalmente concluyentes, sugieren que la especialización de industrias relacionadas, el tamaño de los mercados laborales, el tamaño relativo de los establecimientos manufactureros y las economías de urbanización (para el caso de las grandes ciudades) han sido factores que han influido en el crecimiento del empleo manufacturero urbano en México durante la década de los noventa.

\section{Bibliografía}

Arrow, Kenneth (1962), "The economic implications of learning by doing", Rev. Econ. Studies, núm. 29, pp. 155-173.

Bartik J., Timothy (1985), "Business location decisions in the United States: estimates of the effects of unionization, taxes, and other characteristics of states", Journal of Business and Economics Statistics, vol. 3, núm. 1, 14-22.

Becatinni, Giacomo (1998), Distretti industriali e made in Italy, Ed. Bollati Boringhieri, Torino.

Brown, Flor y Lilia Domínguez (1997), "¿Es posible conformar distritos industriales? La experiencia del calzado en León, Guanajuato", en E. Dussel, Michael Piore y Clemente Ruiz (eds.), Pensar globalmente y actuar regionalmente, Uni- 
versidad Nacional Autónoma de México, Editorial Janus, México.

Carlton W., Dennis (1983), "The location choices of new firms: an econometric model with discrete and continuous endogenous variables", The Review of Economics and Statistics, vol. LXV, núm. 3, 440-449.

Carrillo, Jorge and Alfredo Hualde (2000), "Entorno territorialsectorial. La industria electrónica", en Jorge Carrillo (comp.), Aglomeraciones Locales o Clusters. Evolución Empresarial e Institucional en el Norte de México, El Colegio de la Frontera Norte, Fundación Friederich Ebert, Ensenada.

Dutrénit, Gabriela y Mario Capdeville (1993), "El perfil tecnológico de la industria mexicana y su dinámica innovadora en la década de los ochenta", El Trimestre Económico, julio-septiembre, núm. 239, vol. LX(3), 643-673.

Glaeser, E., H. D. Kallal, J.A. Scheinkman y A. Shleifer (1992), "Growth in cities", Journal of Political Economy, núm. 100, 1126-1152.

Graizbord, Boris y Crecencio Ruiz (1999), "Reestructuración regional sectorial en México, 1980-1993: una evaluación", Comercio Exterior, vol. 49, núm. 4, 321-330.

Hanson, Gordon (1994), "Regional adjustment to trade liberalization", National Bureau of Economic Research Working Papers Series, núm. 4713.

Henderson, Vernon (1974), "The sizes and types of cities", The American Economic Review, vol. LXIV, núm. 4, 640-656.

Jacobs, Jane (1969), The economy of the cities, Vintage Books, New York.

- (1984), Cities and wealth of nations, Vintage Books, Random House, New York.. 
Krugman, Paul y Raúl Livas (1992), "Trade policy and the third world metropolis", National Bureau of Economic Research Working Papers Series, núm. 4238.

Livas, Raúl (1992), "Regional implications of international trade in Mexico", mimeo, MIT.

Marshall, Alfred (1920), Principles of economics, 8th edition reprinted, Macmillan.

Mendoza, Eduardo (1999), "Reubicación trasnacional como impulso a la formación de distritos industriales. El caso de la región Saltillo-Ramos Arizpe", en Clemente Ruiz y Enrique Dussel (comps.), Dinámica regional y competitividad industrial, Jus/UNAM/Fundación Friederich Ebert.

_ (2002), "Agglomeration economies and urban manufacturing growth in the northern border cities of Mexico", Economía Mexicana, vol. XI, núm. 1, pp. 163-190.

Romer, Paul M. (1986), "Increasing returns and long run growth", Journal of Political Economy, vol. 94, 1002-1037.

Tamayo Flores, Rafael (2000), "Location factors and spatial deconcentration of manufacturing growth in Mexico: What do we know and how do we know it", Economía, Sociedad y Territorio, vol. II, núm. 8, El Colegio Mexiquense, 593-640.

Enviado: 13 de mayo de 2002. Reenviado: 23 de septiembre de 2002.

Reenviado: 23 de enero de 2003. Aceptado: 30 de enero de 2003. 